NASA Technical Memorandum 85970

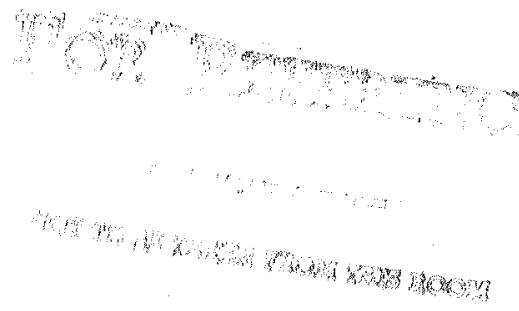

\title{
Improvements in the Accuracy and Stability of Algorithms for the Small-Distrubance and Full-Potential Equations Applied to Transonic Flows
}

Peter M. Goorjian

June 1984

LIRMAMY GOPY

\section{1984}

HNCLEY RESEARCH CENTER

IMBRARY, NASA

UPMPTON, VIRCINA 


\section{Improvements in the Accuracy} and Stability of Algorithms for the Small-Distrubance and Full-Potential Equations Applied to . Transonic Flows

Peter M. Goorjian, Ames. Research Center, Moffett Field, California

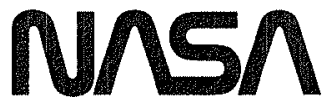

National Aeronautics and

Space Administration 
IMPROVEMENTS IN THE ACCURACY AND STABILITY OF ALGORITHMS FOR THE

SMALL-DISTURBANCE AND FULL-POTENTIAL EQUATIONS

APPLIED TO TRANSONIC FLOWS

Peter M. Goorjian

NASA Ames Research Center

Moffett Field, CA 94035 USA

\section{INTRODUCTION}

This paper describes numerical techniques that improve the accuracy and stability of algorithms for the sma11-disturbance and full-potential equations used to calculate transonic flows. For the small-disturbance equation, the algorithm improvements are: 1) the use of monotone switches in the type-dependent finite-differencing, and 2) the use of stable and simple second-order-accurate spatial differencing. These improvements are for steady and unsteady transonic flows. For the steady, full-potent1al equation, the improvement is in the use of a monotone switch in the type-dependent finte-differencing of an approximate-factorization (AF2) algorithm. All these improvements can be implemented in present computer codes by making minor coding modifications.

\section{SMALL-DISTURBANCE EQUATION IMPROVEMENTS}

\section{Monotone Implicit Algorithms}

Most of the algorithms in transonic small-disturbance potential codes use the MurmanCole [1] method of switching the differencing scheme for regions of supersonic and subsonic flow. A weakness in this method is that it allows stable solutions for flow fields containing entropy-violating expansion shocks in one-dimensional model cases, as shown in Ref. 2. In comparison, the implicit method presented here, which utilizes the monotone Godunov switch [3], does not allow such nonphysical solutions. In two-dimensional flows over airfoils [2], this weakness in the Murman-Cole switch allows numerical instabilities to develop in the calculations near the leading edges of the airfoils. In some cases of steady flows, converged solutions are obtained by the monotone approximate-factorization algorithm (MAF-G) (see Fig. 1 from Ref. 2), whereas calculations using the Murman-Cole switch (AF2) are unstable. In some cases of unsteady flows, the monotone method (LTRAN2-MG) allows the use of larger time-steps than the older method (LTRAN2), which uses the Murman-Cole switch. In Figs. 2(a) and 2 (b) [2], a case is shown in which both methods use the larger time-step and the older method diverges (1.e.', computer overflow); here the older method required a time-step 12 times smallex in order to agree with the monotone method. The jaggedness near the leading edge in the LTRAN2-MG results is due to the use of measured ordinates from an experimental model. This jaggedness provides a severe test case for the stability of any algorithm.

\section{Two-Dimensional Steady Algorithm}

\section{Governing Equation}

The MAF-G algorithm is for the low-frequency, unsteady, sma1l-disturbance potential equation [2]

$$
2 \mathrm{kM}_{\infty}^{2} \phi_{x t}=\left[\left(1-M_{\infty}^{2}\right)-(\gamma+1) M_{\infty}^{m} \phi_{x}\right] \phi_{x x}+\phi_{y y}
$$

Although Eq. (1) is physically meaningful for unsteady flows, MAF-G is nonconservative in time for more efficient calculations of steady flows. The algorithm for unsteady flows, namely, LTRAN2-MG, is presented in detail in Ref. 2; it was used to generate the results shown in Fig. 2 . 


\section{Algorithm}

MAF-G is a modification of an approximate-factorization implicit algorithm, AF2, which uses the Murman-Cole switch. The MAF-G scheme uses the monotone switch of Godunov. It is given by the following two-step, findte-difference approximation to Eq. (1) at mesh point $(i, j)$.

Step 1:

$$
\left[\alpha-\left(A_{1, j} D_{x}\right)\right] f_{1, j}=\left[\alpha^{2} \delta_{x}+\left(A_{1, j} D_{x}\right) \delta_{y y}\right] \phi_{1, j}^{n}+\alpha(\omega-1) R_{1, j}^{n}
$$

Step 2:

$$
\left(\alpha \delta_{x}^{\tilde{y}}-\delta_{y y}\right) \phi_{1, j}^{n+1}=f_{1, j}^{n}
$$

where

$$
\begin{aligned}
& A_{i, j} D_{x}=\tilde{G}_{i, j} \vec{\Delta}_{x}+\hat{G}_{1, j} \overleftarrow{\Delta}_{x} \\
& \tilde{G}_{i, j}=\left(1-\varepsilon_{i, j}\right)\left[\tilde{A}_{i+(1 / 2), j}+\hat{A}_{1-(1 / 2), j}\right]+\tilde{A}_{1-(1 / 2), j} \\
& \hat{G}_{1, j}=\varepsilon_{1-1, j}\left[\tilde{A}_{1-(1 / 2), j}+\hat{A}_{1-(3 / 2), j}\right]+\hat{A}_{1-(1 / 2), j} \\
& \tilde{A}_{i-(1 / 2), j}=\frac{1}{2} C_{1}+C_{2} \tilde{u}_{i-(1 / 2), j}^{n}, \quad \hat{A}_{i-(1 / 2), j}=\frac{1}{2} C_{1}+C_{2} \hat{u}_{i-(1 / 2), j}^{n} \\
& \tilde{u}_{i-(1 / 2), j}^{n}=\bar{u}+\left[1-\varepsilon_{1-(1 / 2), j}\right]\left[u_{i-(1 / 2), j}^{n}-\bar{u}\right] \\
& \hat{u}_{i-(1 / 2), j}^{n}=\bar{u}+\varepsilon_{i-(1 / 2), j}\left[u_{i-(1 / 2), j}^{n}-\bar{u}\right] \\
& C_{1}=1-M_{\infty}^{2}, \quad C_{2}=-\frac{1}{2}(\gamma+1) M_{\infty}^{m}, \quad \bar{u}=-C_{1} / 2 C_{2} \\
& \varepsilon_{i+(1 / 2), j}=\left\{\begin{array}{lll}
0 & \text { if } u_{i+(1 / 2), j} \leq \bar{u} & \text { (subsonic) } \\
1 & \text { otherwise } & \text { (supersonic) }
\end{array}\right. \\
& \varepsilon_{i, j}= \begin{cases}0 & \text { if } \quad u_{1+(1 / 2), j}+u_{1-(1 / 2), j} \leq 2 \bar{u} \text { (possible upstream moving shock) } \\
1 & \text { otherwise (possible downstream moving shock) }\end{cases} \\
& u_{i-(1 / 2), j}^{n}=\delta_{x} \phi_{i, j}^{n}=\frac{\phi_{1, j}^{n}-\phi_{1-1, j}^{n}}{x_{1}-x_{1-1}}, \quad \alpha=2 k_{\infty}^{2} / \Delta t \\
& \overleftarrow{\Delta}_{x} f_{1, j}^{n}=\frac{f_{1, j}^{n}-f_{i-1, j}^{n}}{1 / 2\left(x_{i+1}-x_{1-1}\right)}, \quad \vec{\Delta}_{x} f_{1, j}^{n}=\frac{f_{i+1, j}^{n}-f_{i, j}^{n}}{1 / 2\left(x_{1+1}-x_{i-1}\right)}
\end{aligned}
$$

and where $\omega$ is a relaxation parameter, $R^{n}$ is the residual given by

$$
R_{1, j}^{n}=\left(A_{1, j} D_{x}^{\delta} \delta_{x}+\delta_{y y}\right) \phi_{1, j}^{n}
$$

and $\overline{\mathrm{u}}$ is the sonic value of $\phi_{\mathrm{X}}$. The Murman-Cole switch only uses the $\varepsilon_{1, j}$ switch [2]. The MAF-G scheme is identical to AF2 except in regions where the flow fleld changes type - near the sonic lines and shock waves.

\section{Second-Order-Accurate Supersonic Spatial Differencing}

Current methods for calculating transonic flows with the small-disturbance equation typically are only first-order accurate in the supersonic regions of the flow. However, calculations using the full-potentlal show slgnificant improvements in accuracy when second-order methods are used. In thds paper, a stable, simple algorithm [4] is described that is a second-order-accurate extension of the implicit monotone algorithm described above. For steady flow, Figs. 3(a)-3(c) show calculations of 
flow over a Korn airfoil, for coarse, medium, and fine grids. Note in Fig. $3(a)$ the improvement in resolution with the second-order method. Also, the convergence rates of the two methods are essentially the same. The improvement in the unsteady algorithm (LTRAN2-MG) and improvements in unsteady flow calculations are presented in Ref. 4 .

\section{Algorithm}

The modifications to MAF-G to implement second-order accuracy are made by changing only the first step of MAF-G, given by Eq. (2). Let

$$
A_{i, j}^{\prime} D_{x}^{\prime}=A_{i, j} D_{x}+(\Delta x) \hat{\delta}_{x}^{G_{i, j}^{\prime}} \overleftarrow{\Delta}_{x}
$$

where

$$
\hat{G}_{i, j}^{\prime}=\varepsilon_{1, j} \varepsilon_{1-(1 / 2), j}\left[\hat{A}_{1-(1 / 2), j}+\hat{A}_{i-(3 / 2), j}\right]
$$

and

$$
\alpha^{\prime}=\alpha \Delta x \hat{\delta}_{x} \varepsilon_{1, j} \varepsilon_{i-(1 / 2), j}
$$

Then the modified step 1 is given by the following:

Step 1':

$$
\left[\left(\alpha+\alpha^{\prime}\right)-\left(A_{1, j}^{\prime} D_{x}^{\prime}\right)\right] f_{1, j}=\left[\alpha\left(\alpha+\alpha^{\prime}\right) \delta_{x}^{\xi}+\left(-\alpha^{\prime}+A_{1, j}^{\prime} D_{x}^{\prime}\right) \delta_{y y}\right] \phi_{1, j}^{n}+\alpha(\omega-1) R_{1, j}^{\prime n}
$$

Now the residual is given by

$$
R_{i, j}^{\prime n}=\left(A_{i, j}^{\prime} D_{x}^{\prime} \delta_{x}+\delta_{y y}\right) \phi_{i, j}^{n}
$$

\section{FULL-POTENTIAL EQUATION IMPROVEMENT}

\section{Monotone Implicit Algorithm}

Most of the algorithms in transonic, full-potential codes use type-dependent differencing that is a generalization of the Murman-Cole switch, such as the method of Jameson and the AF2 method of Holst and Ballhaus [5]. These methods suffer from a weakness similar to that of the Murman-Cole switch in dealing with nonphysical expan slons shocks. A monotone implicit method (AF2) has been developed [2] that eliminates this deficiency at sonic expansion points, as demonstrated by the calculations shown in Figs. 4(a) and 4(b) [2] for a two-dimensional case of flow over a modified, double-wedge profile. The full details of the MAG algorithm are given in Ref. 2 . Here we describe the essence of the new switch. The AF2 method uses a nonmonotone switch, called upwind-density biasing, to stabilize the calculations in supersonic regions of the flow. That switch is implemented in the mass flux. An example, for $\rho \phi_{X}$, is

$$
\bar{\rho}_{1+(1 / 2), j} \delta_{x} \phi_{1, j}^{n}=\left\{\rho_{1+(1 / 2), j}-\nu_{1, j}\left[\rho_{1+(1 / 2), j}-\rho_{1-(1 / 2), j}\right]\right\} \vec{\delta}_{x} \phi_{i, j}
$$

MAF employs a monotone swltch based on Godunov's ideas. An example, for $\rho \phi_{x}$, is

$$
\begin{aligned}
\bar{\rho}_{1+(1 / 2), j} \delta \phi_{x} \mathbf{n}= & \left\{\rho_{1+(1 / 2), j}-\bar{v}_{1+(1 / 2), j}\left[\rho_{1+(1 / 2), j}-\rho \star\right]\right\} \vec{\delta}_{x} \phi_{1, j} \\
& +\left\{\hat{v}_{1-(1 / 2), j}\left[\rho_{1-(1 / 2), j}-\rho^{*}\right]\right\} \vec{\delta}_{\mathbf{x}} \phi_{1, j}
\end{aligned}
$$

The essence of the difference between MAF and AF2 is seen by comparing Eqs. (5) and (6). Whereas Eq. (5) uses the switch $v$ to smoothly shift the flow variables upwind as the flow becomes supersonic, Eq. (6) uses the switches $\tilde{v}$ and $\hat{v}$ to smoothly shift the flow variables to sonic values $\rho^{*}$ and $q^{*}$, and to shift flow variables located upwind away from sonic values. Both methods use rotated differencing and treat shock waves identically [2], but the monotone method is more stable at sonic expansion points. 


\section{REFERENCES}

1. Murman, E. M. and Cole, J. D., "Calculations of Plane Steady Transonic Flow," AIAA Journal, Vo1. 9, No. 2, 1971, pp. 114-121.

2. Goorjian, P. M., Meagher, M. E., and Van Buskirk, R., "Monotone Implicit Algorithms for the Sma11-Disturbance and Ful1-Potential Equations Applied to Transonic Flows," AIAA Paper 83-0371, Reno, Nev., 1983.

3. Godunov, S. K., "A Finite-Difference Method for Inviscid Transonic Flows with Embedded Shock Waves," Mat. Sb. 47, 1959, p. 271; also: Cornell Aeronautical Lab. (Calspan) Translation.

4. Goorfian, P. M., and Van Buskirk, R., "Second Order Accurate Supersonic Differencing for the Sma11-Disturbance Potential Equations Applied to Transonic Flows," AIAA Paper 84-0091, Reno, Nev., 1984.

5. Holst, T. I. and Ballhaus, W. F., "Fast, Conservative Scheme for the Ful1Potential Equation Applied to Transonic Flows," AIAA Journal, Vol. 17, Feb. 1979, pp.

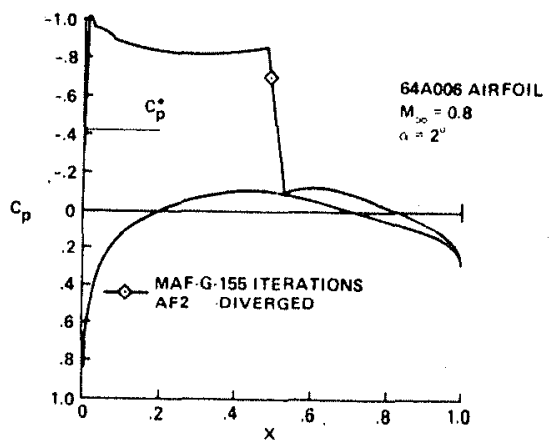

Fig. I Converged solution obtained from algorithm using the monotone Godunov switch MAF-G; algorithm using nonmonotones switch AF2 diverged.

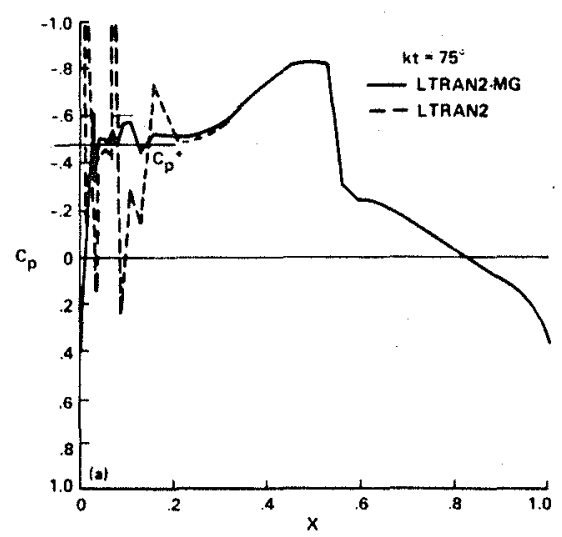

a) $\mathrm{kt}=75^{\circ}$

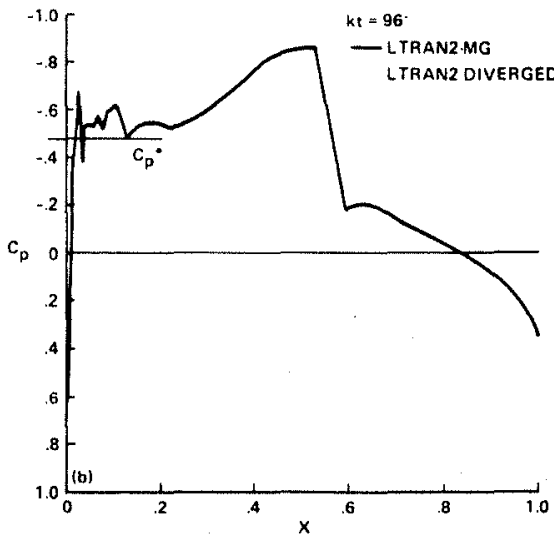

b) $\mathrm{kt}=96^{\circ}$

Fig. 2 Comparison of algorithms using the monotone Godunov switch LTRAN2-MG and nonmonotone switch LTRAN2. Plots of upper-surface pressure coefficients of a NACA 64A010 airfoil (experimental mode1 ordinates) in pitching motion. Time given in degrees of motion $k t$ and $M_{\infty}=0.80$. 


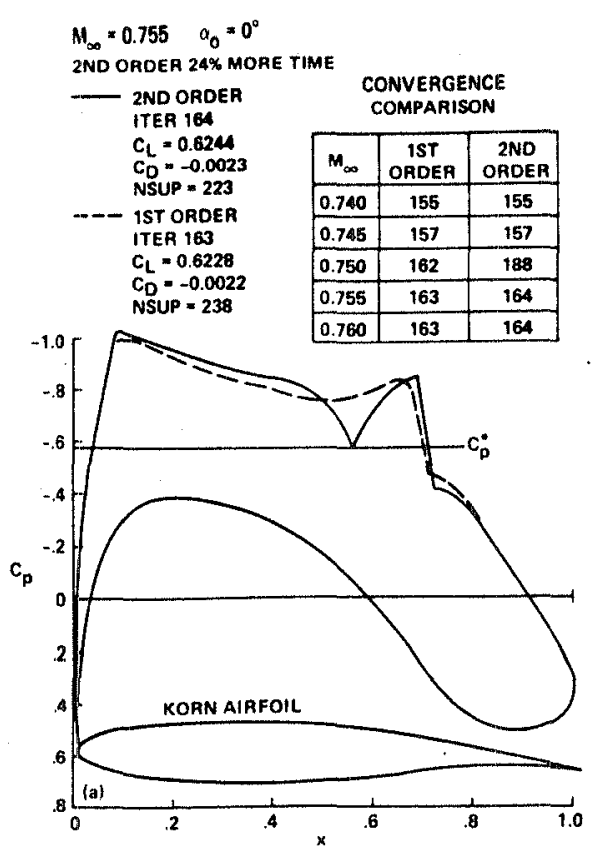

a) Coarse grid

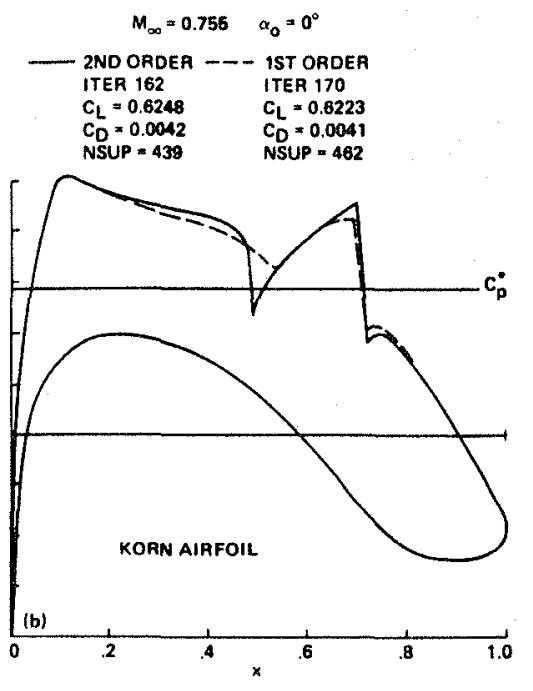

b) Median grid

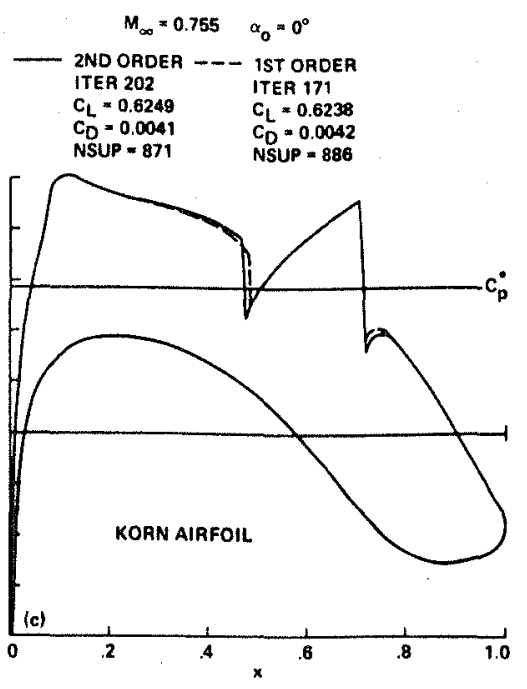

c) Fine grid

Fig. 3 Comparison of first- and second-order methods for steady flow over a Korn airfoil; pressure-coefficients plot: $M_{\infty}=0.755$ and $\alpha_{0}=0$.

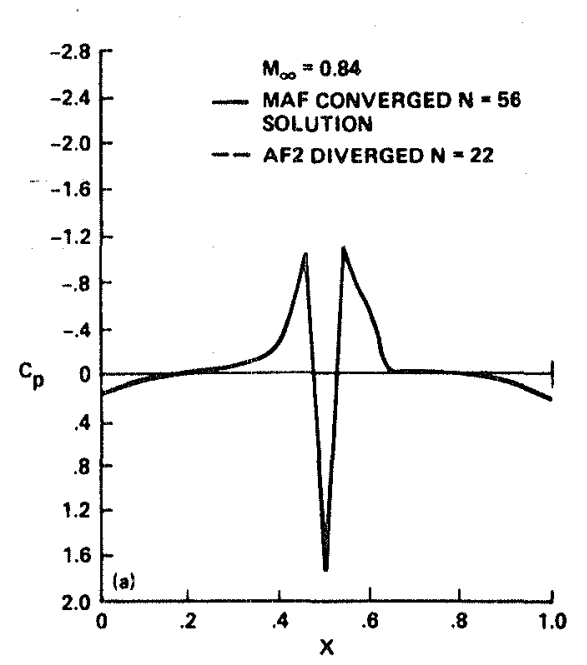

a) Converged solution using MAF, nonmonotone algorithm diverged.
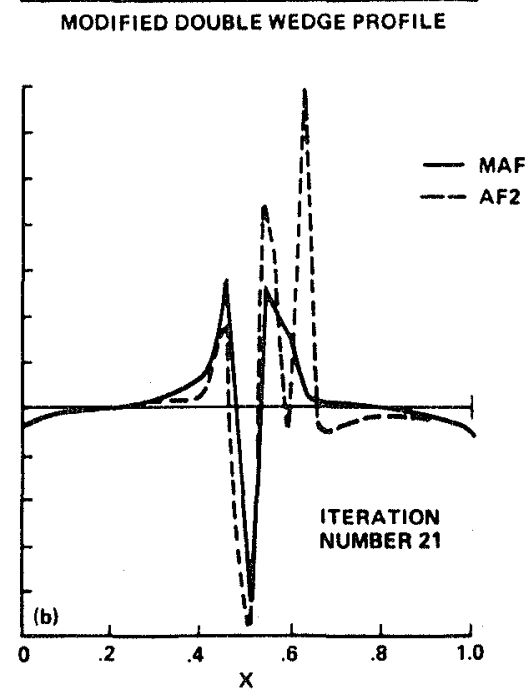

b) Iteration No. 21 .

Fig. 4 Comparison of monotone MAF and nonmonotone AF2 algorithms for flow over a modified, double-wedge profile. 


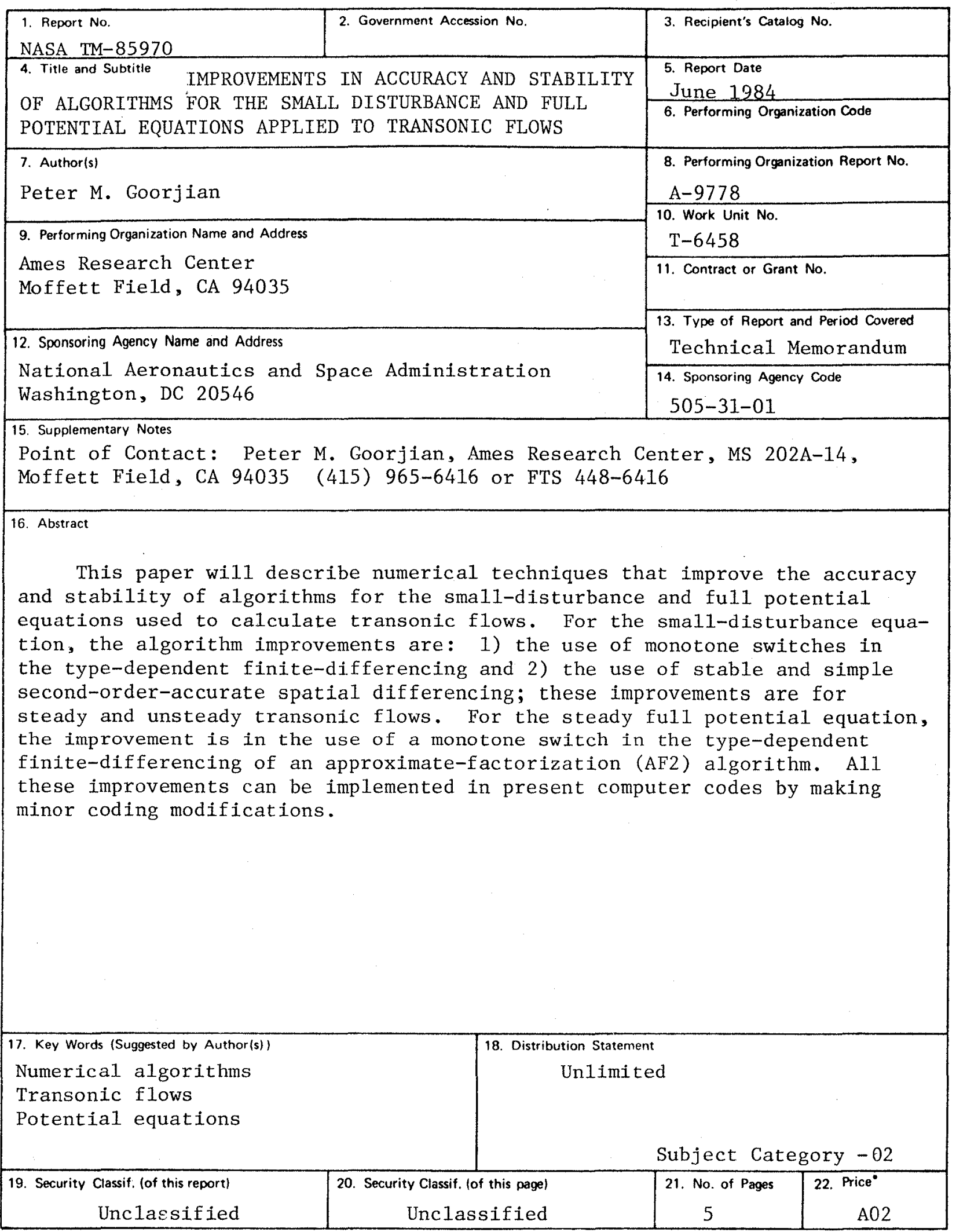

"For sale by the National Technical Information Service, Springfield, Virginia 22161 
End of Document 